\title{
Taming the Wrapping of Integer Arithmetic
}

\author{
Axel Simon and Andy King \\ Computing Laboratory, University of Kent, Canterbury, UK \\ \{a.simon, a.m.king\}@kent.ac.uk
}

\begin{abstract}
Variables in programs are usually confined to a fixed number of bits and results that require more bits are truncated. Due to the use of 32-bit and 64-bit variables, inadvertent overflows are rare. However, a sound static analysis must reason about overflowing calculations and conversions between unsigned and signed integers; the latter remaining a common source of subtle programming errors. Rather than polluting an analysis with the low-level details of modelling two's complement wrapping behaviour, this paper presents a computationally light-weight solution based on polyhedral analysis which eliminates the need to check for wrapping when evaluating most (particularly linear) assignments.
\end{abstract}

\section{Introduction}

Static analysis methods are increasingly used to prove the partial correctness of software [5]. In contrast to formal methods that verify properties of a highlevel specification, a static analysis is complicated by low-level details of source code. For instance, while a specification expresses properties over arbitrary integers, variables in a program are usually confined to finite integer types that are deemed to be large enough to hold all values occurring at run-time. On one hand, the use of 32-bit and 64-bit variables make accidental overflows rare and adding checks to each transfer function of the analysis seems to be excessive considering the infrequency of variable overflows. On the other hand, programmers often inadvertently introduce wrapping when converting between signed and unsigned variables and deliberately exploit the wrapping effects of two's complement arithmetic. Thus, wrapping itself should not be considered harmful, particularly when the objective of an analysis is the verification of a different property, such as absence of out-of-bound memory accesses [8,9].

In fact, there is a danger in flagging all wrapping, since any intentional use of wrapping generates a warning message which the developer immediately dismisses as a false positive. The code in Figure 1 illustrates why this is a problem. The purpose of the shown $\mathrm{C}$ function is to print how many times each individual character occurs in the given string *str. To this end, the elements of dist are initialised to zero by the call to memset. In the loop that follows, the $n$th element is incremented each time a character $n$ is encountered in str. The for loop then prints the distribution of printable ASCII characters.

The shown program is correct on platforms where char is unsigned such as Linux on PowerPC. However, for Linux on x86 and MacOS X on PowerPC, char 


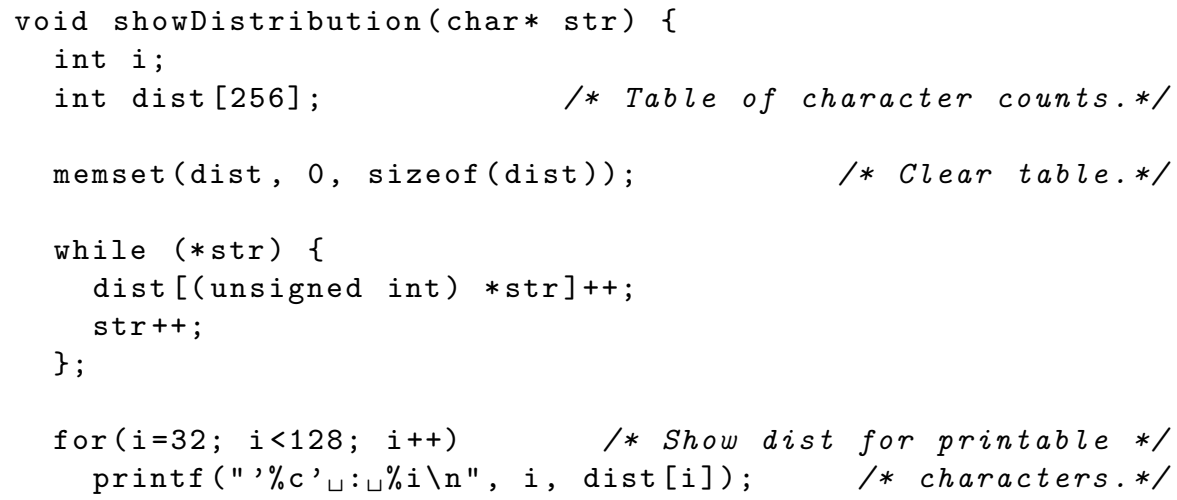

Fig. 1. Example C function that counts the occurrences of each character.

is signed. In the latter case, the value $*$ str which is used to index into dist can take on values in the range $[-128,127]$. Although the programmer intended to convert the value of $*$ str to an unsigned value before the extension to a 4 -byte quantity takes place, the $\mathrm{C}$ standard [3] dictates that the value of $*$ str is first promoted to int before the conversion to an unsigned type is performed. Hence, dist can be accessed at indices $\left[2^{32}-128 \ldots 2^{32}-1\right] \cup[0 \ldots 127]$, of which the first range is out-of-bounds. A static analysis that considers all wrapping to be erroneous would flag this statement as possibly faulty. However, since the programmer expects that wrapping does occur (namely when converting from a char to an unsigned quantity), the warning about wrapping at the unsigned int-level will be dismissed as a false positive. Hence, the analysis above should flag the out-of-bound array access but treat wrapping itself as intentional.

In this work, we propose a re-interpretation of polyhedra in which the wrapping of integer calculations is reflected in the classic polyhedral domain [6]. In particular, we avoid making cross-cutting changes to all transfer functions but refine the approximation relation such that wrapping is mostly implicit, that is, the need for extra polyhedral operations is largely finessed. For the few cases in which wrapping has to be addressed in the transfer functions, we illustrate how to wrap values within the polyhedral domain and propose an algorithm for doing so. To summarise our contributions, this paper presents:

- an approximation relation that implicitly wraps polyhedral variables;

- an algorithm to perform wrapping in the polyhedral domain;

- a formal description of an analysis that faithfully models wrapping and an accompanying correctness argument.

We commence with the definition of a small language and its concrete semantics. Section 3 introduces polyhedral analysis. Sections 4 and 5 explain how wrapping is supported. Section 6 presents a wrapping-aware polyhedral analysis which is discussed in Section 7. We conclude with the related work in Section 8. 


\section{A Language Featuring Finite Integer Arithmetic}

The function shown in Figure 1 demonstrates that it is important to clarify where wrapping can arise in a program. This is particularly true when arguing the correctness of an analysis. To this end we introduce the language $\mathcal{L}$ (ELang) which is a subset of an intermediate language which is used to analyse $\mathrm{C}$ programs.

\subsection{The Syntax of $\mathcal{L}$ (ELang)}

$\mathcal{L}$ (ELang) features linear expressions and casts between integers. In the following grammar of $\mathcal{L}$ (ELang), $(\mathrm{T})^{*}$ denotes the repetition of the non-terminal $\mathrm{T}$.

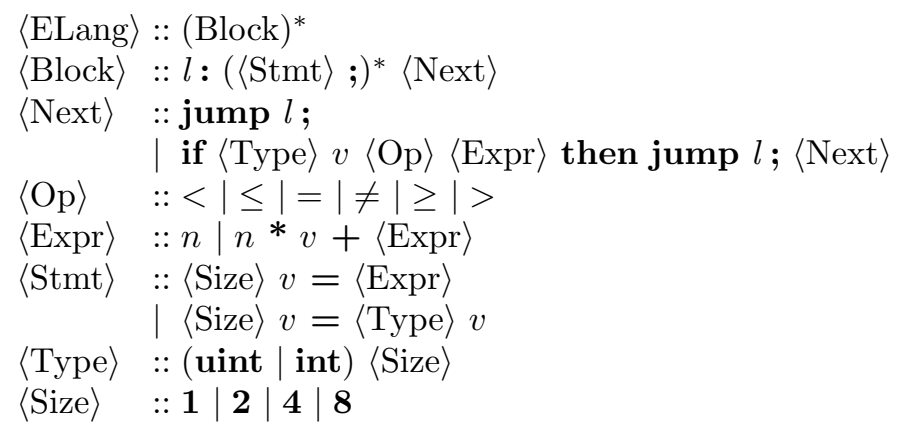

An ELang program consists of a sequence of basic blocks with execution commencing with the first block. Each basic block consists of a sequence of statements and a list of control-flow instructions. In the sequel, we use lookupBlock $(l)$ and lookupNext $(l)$ to access the statements and control-flow instructions, respectively, of basic block $l$. The control-flow of a basic block consists of either a jump statement or a conditional which itself is followed by more control flow instructions. The $\langle$ Stmt $\rangle$ production is restricted to the two statements of interest, namely the assignment of linear expressions to a variable and a type cast. We require that each variable in the program is used with only one size which is always specified in bytes. In particular, the assignment statement and the conditional require that all occurring variables are of the same size. Note, though, that variables may be used as an uint (unsigned integer) in one statement and as an int (signed integer) in another.

\subsection{The Semantics of $\mathcal{L}$ (ELang)}

In order to specify the semantics of $\mathcal{L}$ (ELang), we introduce the following notation. Let $\mathbb{B}=\{0,1\}$ denote the set of Booleans. A vector $\boldsymbol{b}=\left\langle b_{w-1}, \ldots b_{0}\right\rangle \in \mathbb{B}^{w}$ is interpreted as unsigned integer by $v a l^{w, \text { uint }}(\boldsymbol{b})=\sum_{i=0}^{w-1} b_{i} 2^{i}$ and as signed integer by val $^{w, \text { int }}(\boldsymbol{b})=\left(\sum_{i=0}^{w-2} b_{i} 2^{i}\right)-b_{w-1} 2^{w-1}$. Conversely, bin ${ }^{w}: \mathbb{Z} \rightarrow \mathbb{B}^{w}$ converts an integer to the lower $w$ bits of its Boolean representation. Formally, $\operatorname{bin}^{w}(v)=\boldsymbol{b}$ iff there exists $\boldsymbol{b}^{\prime} \in \mathbb{B}^{q}$ such that $v a l^{(q+w), \text { int }}\left(\boldsymbol{b}^{\prime} \| \boldsymbol{b}\right)=v$ where $\|$ denotes the concatenation of bit-vectors. For instance, $\operatorname{bin}^{3}(15)=\langle 1,1,1\rangle$ since 
Basic Blocks.

$\llbracket l: s_{1} ; \ldots s_{n} ; \rrbracket_{\text {Block }}^{\natural} \sigma=\llbracket \operatorname{lookupNext}(l) \rrbracket_{\text {Next }}^{\natural}\left(\llbracket s_{n} \rrbracket_{\text {Stmt }}^{\natural}\left(\ldots\left(\llbracket s_{1} \rrbracket_{\text {Stmt }}^{\natural} \sigma\right) \ldots\right)\right)$

Control Flow.

$\llbracket$ jump $l \rrbracket_{\text {Next }}^{\natural} \sigma=\llbracket \operatorname{lookupBlock}(l) \rrbracket_{\text {Block }}^{\natural} \sigma$

【if $t s v$ op exp then jump $l ; n x t \rrbracket_{\mathrm{Next}}^{\natural} \sigma=$

$\begin{cases}\llbracket \operatorname{lookupBlock}(l) \rrbracket_{\mathrm{Block}}^{\natural} \sigma & \text { if } v_{a l}^{8 s, t}\left(\sigma^{s}\left(a d d r^{\natural}(v)\right)\right) \text { op } \operatorname{val}^{8 s, t}\left(\llbracket \exp \rrbracket_{\mathrm{Expr}}^{\natural, s} \sigma\right) \\ \llbracket n x t \rrbracket_{\text {Next }}^{\natural} \sigma & \text { otherwise }\end{cases}$

Expressions.

$\llbracket n \rrbracket_{\operatorname{Expr}}^{\natural, s} \sigma=\operatorname{bin}^{8 s}(n)$

$\llbracket n * v+\exp \rrbracket_{\mathrm{Expr}}^{\natural, s} \sigma=\operatorname{bin}^{8 s}(n) *^{8 s} \sigma^{s}\left(a d d r^{\natural}(v)\right)+{ }^{8 s} \llbracket \exp \rrbracket_{\mathrm{Expr}}^{\natural, s} \sigma$

Assignment.

$\llbracket s v=\exp \rrbracket_{\mathrm{Stmt}}^{\natural} \sigma=\sigma\left[a d d r^{\natural}(v) \stackrel{s}{\mapsto} \llbracket \exp \rrbracket_{\mathrm{Expr}}^{\natural, s} \sigma\right]$

Type Casts.

$\llbracket s_{1} v_{1}=t s_{2} v_{2} \rrbracket_{\text {Stmt }}^{\natural} \sigma=\sigma\left[a d d r^{\natural}\left(v_{1}\right) \stackrel{s_{1}}{\mapsto} \operatorname{bin}^{8 s_{1}}\left(v a l^{8 s_{2}, t}\left(\sigma^{s_{2}}\left(a d d r^{\natural}\left(v_{2}\right)\right)\right)\right)\right]$

Fig. 2. Concrete Semantics of $\mathcal{L}$ (ELang).

$v a l^{5, \text { int }}(\langle 0,1,1,1,1\rangle)=15$. In order to distinguish calculations on Boolean vectors from standard arithmetic, let $+^{w}, *^{w}: \mathbb{B}^{w} \times \mathbb{B}^{w} \rightarrow \mathbb{B}^{w}$ denote addition and multiplication that truncate the result to the lower $w$ bits, for instance $\langle 1,1,1,1\rangle+{ }^{4}\langle 0,0,0,1\rangle=\langle 0,0,0,0\rangle$. Note that the signedness of the arguments of $+^{w}$ and $*^{w}$ do not affect the result of these operations.

$\mathcal{L}$ (ELang) programs operate in a virtual memory environment which we formalise as a sequence of bytes. Let $\mathcal{B}=\mathbb{B}^{8}$ denote the set of bytes and $\Sigma=\mathcal{B}^{2^{32}}$ all states of 4 GByte that a program on a 32-bit architecture can take on. Let $\sigma \in \Sigma$ denote a given memory state of a program and let $\sigma^{s}:\left[0,2^{32}-1\right] \rightarrow \mathcal{B}^{s}$ denote a read access at the given 32-bit address where $s \in\{1,2,4,8\}$ is the number of bytes to be read. A write operation is formalised as a substitution $\sigma[a \stackrel{s}{\mapsto} v]$. The resulting store $\sigma^{\prime}=\sigma[a \stackrel{s}{\mapsto} v]$ satisfies $\sigma^{\prime s}(a)=v$ and furthermore $\sigma^{\prime 1}(b)=\sigma^{1}(b)$ for all addresses $b \notin\{a, \ldots a+s-1\}$.

Figure 2 presents the concrete semantics (or natural semantics, hence the $\downarrow$ ) of the $\mathcal{L}$ (ELang) language. These definitions use $a d d r^{\natural}(v) \in\left[0,2^{32}-1\right]$ which maps the program variable $v$ to its address in memory. We assume that $a d d r^{\natural}$ maps different variables to non-overlapping memory regions, an assumption that makes $\mathcal{L}$ (ELang) independent of the endianness of an architecture.

The concrete semantics manipulates the store mainly by operations on bitvectors; only in the conditional and in the cast are bit vectors interpreted as numbers. In these cases the signedness of the variables can actually influence the result. In particular, the type $t$ of the cast determines if the source bit-vector is sign-extended (if $t=$ int) or zero-extended (if $t=$ uint) when $s_{1} \geq s_{2}$. We now proceed by abstracting this semantics so as to specify a polyhedral analysis. 


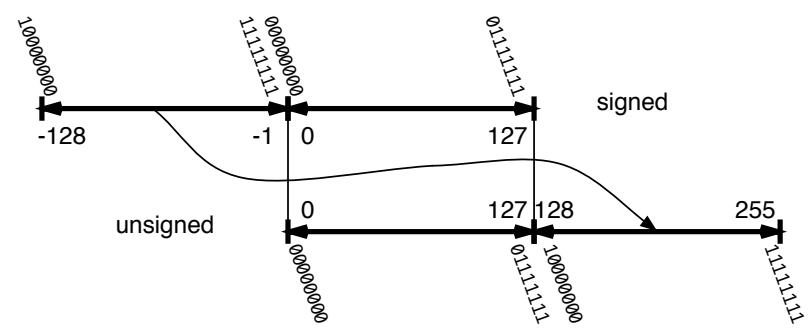

Fig. 3. The difference between a signed and an unsigned access can be interpreted as a wrap of negative values to the upper range in an unsigned access.

\section{Polyhedral Analysis of Finite Integers}

Two's complement arithmetic exploits the wrapping behaviour of integer variables that are confined to a fixed number of bits. For instance, subtracting 1 from an integer is equivalent to adding the largest representable integer value. In fact, the binary representation of the signed integer -1 is identical to that of the largest, unsigned integer of the same size. In the context of verification, this dichotomy in interpretation cannot be dismissed since $\mathcal{L}$ (ELang) has insufficient information about the signedness of assignments. This omission allows our model to be applied to languages which freely mix signed and unsigned values, e.g. C.

Accessing the same bit sequence as either signed or unsigned integer corresponds to a wrapping behaviour in that the negative range of the signed integer wraps to the upper range of an unsigned integer, see Figure 3. This wrapping behaviour of finite integers creates a mismatch against the infinite range of polyhedral variables. We present our solution to this mismatch in two parts: Section 4 presents a concretisation map between the polyhedral domain and the bit-level representation of variables. This map wraps values of abstract variables implicitly to finite sequences of bits, thereby alleviating the need to check for wrapped values each time a variable is read or written. In contrast, Section 5 details an algorithm that makes the wrapping of program variables explicit in the abstract domain which is important for casts and the conditional statement whose semantics depend on the size and signedness of the operands.

\subsection{The Domain of Closed, Convex Polyhedra}

In order to make the paper self-contained, this section gives a concise introduction to the notation used in our polyhedral analysis. Let $X=\left\{x_{1}, \ldots x_{n}\right\}$ be a finite set of variables, let $\boldsymbol{x}=\left\langle x_{1}, \ldots x_{n}\right\rangle$ and let $I$ be the set of linear inequalities that can be rewritten as $\boldsymbol{c} \cdot \boldsymbol{x} \leq d$ where $\boldsymbol{c} \in \mathbb{Z}^{n}$ and $d \in \mathbb{Z}$. For brevity we write $e_{1}=e_{2}$ to denote the set of inequalities $\left\{e_{1} \leq e_{2}, e_{2} \leq e_{1}\right\}$ where $e_{i}$ is any linear expression. Furthermore, let $e_{1}<e_{2}$ abbreviate $e_{2} \leq e_{2}-1$. Let $\iota \in I$ be an inequality that can be transformed into $\boldsymbol{c} \cdot \boldsymbol{x} \leq d$, then $\llbracket \iota \rrbracket=\left\{\boldsymbol{x} \in \mathbb{R}^{n} \mid \boldsymbol{c} \cdot \boldsymbol{x} \leq d\right\}$ denotes the induced half-space. Given a finite set of inequalities $E=\left\{\iota_{1}, \ldots \iota_{n}\right\} \subseteq I$ 
the notation $\llbracket E \rrbracket=\bigcap_{i=1, \ldots . n} \llbracket \iota_{i} \rrbracket$ denotes the induced convex polyhedron. Let Poly be the set of all convex polyhedra (polyhedra for short). Given two polyhedra $P_{1}, P_{2} \in$ Poly, let $P_{1} \sqsubseteq P_{2}$ iff $P_{1} \subseteq P_{2}$ and let $P_{1} \sqcap P_{2}=P_{1} \cap P_{2}$. Note that $P_{1} \sqcap P_{2}=\llbracket E_{1} \cup E_{2} \rrbracket$ where $P_{i}=\llbracket E_{i} \rrbracket$ and $i=1,2$. We write $P_{1} \sqcup P_{2}$ to denote the closure of the convex hull [6] of two polyhedra $P_{1}$ and $P_{2}$ which is defined as the smallest polyhedron $P$ such that $P_{1} \cup P_{2} \sqsubseteq P$. Let $P \in P$ oly be non-empty and $V_{i}=\left\{v_{i} \mid\left\langle v_{0}, \ldots v_{n}\right\rangle \in P\right\}$. We write $P(x)=[l, u]$ where $l=\left\lceil\min \left(V_{i}\right)\right\rceil$ if the minimum exists, otherwise $l=-\infty$ and $u=\left\lfloor\max \left(V_{i}\right)\right\rfloor$ if the maximum exists, otherwise $u=\infty$. Define $\exists_{x_{i}}:$ Poly $\rightarrow$ Poly such that $\exists_{x_{i}}(P)=\left\{\left\langle v_{1}, \ldots, v_{i-1}, x, v_{i+1}, \ldots v_{n}\right\rangle \mid\left\langle v_{1}, \ldots v_{n}\right\rangle \in P \wedge x \in \mathbb{R}\right\}$. Let $T$ denote an additional set of temporary variables such that $T \cap X=\emptyset$. All operations above lift from $X$ to $T \cup X$. The operation $P \triangleright x:=e \in P$ oly denotes an update of a variable $x \in X$ to the linear expression $e$ and is defined as $\exists_{t}\left(\llbracket x=t \rrbracket \sqcap \exists_{x}(\llbracket t=e \rrbracket \sqcap P)\right)$ where $t \in T$ does not occur in $e$ and $t \neq x$. The rationale for assigning the result to a temporary variable is that $e$ might contain $x$. Assigning $e$ to a fresh variable $t$ will retain the value of $x$ before it is projected out. If $x$ does not occur in $e$, the update above is equivalent to $\exists_{x}(P) \sqcap \llbracket x=e \rrbracket$. In order to argue about updates of polyhedra, observe the following:

Lemma 1. Let $P \in$ Poly and $P^{\prime}=P \triangleright x_{i}:=\boldsymbol{c} \cdot \boldsymbol{x}+d$ with $\boldsymbol{c} \in \mathbb{Z}^{n}$ and $d \in \mathbb{Z}$. Then $P^{\prime}=\left\{\left\langle v_{1}, \ldots v_{i-1}, v_{i}^{\prime}, v_{i+1}, \ldots v_{n}\right\rangle \mid \boldsymbol{v}=\left\langle v_{1}, \ldots v_{n}\right\rangle \in P \wedge v_{i}^{\prime}=\boldsymbol{c} \cdot \boldsymbol{v}+d\right\}$.

\section{Implicit Wrapping of Polyhedral Variables}

This section formalises the relationship between polyhedral variables and bitsequences that constitute the program state. For simplicity, we assume a one-toone correspondance between the variable names in the program and the polyhedral variables that represent their values. The values of a program variable are merely bit sequences that are prescribed by the possible values of the polyhedral variable. To illustrate, suppose that $\mathrm{x}$ is of type char and $P(x)=[-1,2]$. The represented bit patterns are 11111111, 00000000, 00000001 and 00000010, no matter whether $\mathrm{x}$ is signed or unsigned. These bit patterns are given by $\operatorname{bin}^{8 s}(v)$ which turns a value $v \in[-1,2]$ into a bit sequence of $s$ bytes. Going further, the function bits $s_{a}^{s}: \mathbb{Z} \rightarrow \mathcal{P}(\Sigma)$ produces all concrete stores in which $8 s$ bits at address $a=a d d r^{\natural}(\mathrm{x})$ are set to the value corresponding to $v \in P(x)$ as follows:

$$
\operatorname{bits}_{a}^{s}(v)=\left\{\left\langle r_{8 * 22^{32}} \ldots r_{8(a+s)}\right\rangle\left\|\operatorname{bin}^{8 s}(v)\right\|\left\langle r_{8 a-1} \ldots r_{0}\right\rangle \mid r_{i} \in \mathbb{B}\right\}
$$

Note that this definition only considers the lower $8 s$ bits of the value $v$. For instance, bits $_{a}^{1}(0)=$ bits $_{a}^{1}(256)$ since the lower eight bits of 0 and 256 are equal. The mapping bits $s_{a}^{s}$ can be lifted from the value $v$ of a single variable to the values $\left\langle v_{1}, \ldots v_{n}\right\rangle \in \mathbb{Z}^{n}$ of a vector of variables $\left\langle x_{1}, \ldots x_{n}\right\rangle$, resulting in the stores $\bigcap_{i \in[1, n]} b i t s_{a_{i}}^{s_{i}}\left(v_{i}\right)$. Here $a_{i} \in\left[0,2^{32}-1\right]$ denotes the address of the variable $x_{i}$ in the concrete store and $s_{i} \in \mathbb{N}$ denotes its size in bytes. Observe that, if variables were allowed to overlap, the above intersection might incorrectly collapse to the 
empty set for certain vectors $\left\langle v_{1}, \ldots v_{n}\right\rangle \in \mathbb{Z}^{n}$. Using this lifting, a polyhedron is now related to a set of stores by $\gamma_{\boldsymbol{a}}^{\boldsymbol{s}}:$ Poly $\rightarrow \mathcal{P}(\Sigma)$ which is defined as

$$
\gamma_{\boldsymbol{a}}^{\boldsymbol{s}}(P)=\bigcup_{\boldsymbol{v} \in P \cap \mathbb{Z}^{n}}\left(\bigcap_{i \in[1, n]} \operatorname{bits}_{a_{i}}^{s_{i}}\left(v_{i}\right)\right)
$$

where $\boldsymbol{s}=\left\langle s_{1}, \ldots s_{n}\right\rangle, \boldsymbol{a}=\left\langle a_{1}, \ldots a_{n}\right\rangle$ and $\boldsymbol{v}=\left\langle v_{1}, \ldots v_{n}\right\rangle$.

The definition of $\gamma_{\boldsymbol{a}}^{\boldsymbol{s}}$ provides a criterion for judging the correctness of an abstract semantics. In addition, $\gamma_{\boldsymbol{a}}^{\boldsymbol{s}}$ permits linear expressions to be evaluated in the abstract semantics without the need to address overflows since $\gamma_{\boldsymbol{a}}^{\boldsymbol{s}}$ maps the result of calculations in the polyhedral domain to the correctly wrapped result in the actual program. This property is formalised below:

Proposition 1. Let $e \in \mathcal{L}($ Expr $)$ and $e \equiv \boldsymbol{c} \cdot \boldsymbol{x}+d$, that is, e is a reformulation of $\boldsymbol{c} \cdot \boldsymbol{x}+d$. If $\sigma \in \gamma_{\boldsymbol{a}}^{\boldsymbol{s}}(P)$ then $\sigma\left[a_{i} \stackrel{s_{i}}{\mapsto} \llbracket e \rrbracket_{\text {Expr }}^{\natural, s_{i}}\right] \in \gamma_{\boldsymbol{a}}^{\boldsymbol{s}}\left(P \triangleright x_{i}:=\boldsymbol{c} \cdot \boldsymbol{x}+d\right)$.

Proof. Define $\pi_{i}\left(\left\langle x_{1}, \ldots x_{i-1}, x_{i}, x_{i+1}, \ldots x_{n}\right\rangle\right)=x_{i}$. Since $\sigma \in \gamma_{\boldsymbol{a}}^{\boldsymbol{s}}(P)$ there exists $\boldsymbol{v} \in P \cap \mathbb{Z}^{n}$ such that $\sigma=\bigcap_{i \in[1, n]}$ bits $_{a_{i}}^{s_{i}}\left(\pi_{i}(\boldsymbol{v})\right)$. Let $P^{\prime}=P \triangleright x_{i}:=\boldsymbol{c} \cdot \boldsymbol{x}+d$ for some $\boldsymbol{c} \in \mathbb{Z}^{n}$ and $d \in \mathbb{Z}$. By Lemma 1 , there exists $\boldsymbol{v}^{\prime} \in P^{\prime}$ with $\pi_{j}\left(\boldsymbol{v}^{\prime}\right)=\pi_{j}(\boldsymbol{v})$ for all $j \neq i$. Since $\left\{a_{i}, \ldots a_{i}+s_{i}-1\right\} \cap\left\{a_{j}, \ldots a_{j}+s_{j}-1\right\}=\emptyset$ for all $j \neq i$, there exists $\sigma^{\prime} \in \gamma_{\boldsymbol{a}}^{\boldsymbol{s}}\left(P^{\prime}\right)$ such that $\sigma^{\prime 1}(a)=\sigma^{1}(a)$ for $a \in\left[0,2^{32}-1\right] \backslash\left\{a_{i}, \ldots a_{i}+s_{i}-1\right\}$. Furthermore, the lemma states that $\pi_{i}\left(\boldsymbol{v}^{\prime}\right)=\boldsymbol{c} \cdot \boldsymbol{v}+d$ and, by the definition of $\gamma_{\boldsymbol{a}}^{\boldsymbol{s}}$, it follows that $\sigma^{\prime s_{i}}\left(a_{i}\right)=\operatorname{bin}^{8 s_{i}}(\boldsymbol{c} \cdot \boldsymbol{v}+d)$. To show that $\sigma^{\prime s_{i}}\left(a_{i}\right)=\llbracket e \rrbracket_{\text {Expr }}^{\natural, s_{i}} \sigma$, we find $\boldsymbol{a} \in \mathbb{Z}^{n}, d \in \mathbb{Z}$ such that $e \equiv \boldsymbol{c} \cdot \boldsymbol{x}+d$ and $\llbracket e \rrbracket_{\mathrm{Expr}}^{\natural, s_{i}} \sigma=\operatorname{bin}^{8 s_{i}}(\boldsymbol{c} \cdot \boldsymbol{v}+d)$ by induction over $e$ :

1. Let $e=n$. By definition of $\llbracket \cdot \rrbracket_{\text {Expr }}^{\natural, s}, \llbracket n \rrbracket_{\text {Expr }}^{\natural, s_{i}} \sigma=b i n^{8 s_{i}}(n)=b i n^{8 s_{i}}(\boldsymbol{c} \cdot \boldsymbol{v}+d)$ where $d=n$ and $\boldsymbol{c}=\langle 0, \ldots 0\rangle$. Hence $e \equiv \boldsymbol{c} \cdot \boldsymbol{x}+d$.

2. Let $e=n * x_{j}+e^{\prime}$. Suppose that $\llbracket e^{\prime} \rrbracket_{\mathrm{Expr}}^{\natural, s_{i}} \sigma=\operatorname{bin}^{8 s_{i}}\left(\boldsymbol{c}^{\prime} \cdot \boldsymbol{v}+d^{\prime}\right)$ where $e^{\prime} \equiv \boldsymbol{c}^{\prime} \cdot \boldsymbol{x}+d^{\prime}$. By the definition of $\llbracket \cdot \rrbracket_{\text {Expr }}^{\natural, s}, \llbracket n * x_{j}+e \rrbracket_{\text {Expr }}^{\natural, s_{i}} \sigma=$ $\operatorname{bin}^{8 s}(n) *^{8 s_{i}} \sigma^{s_{i}}\left(a_{j}\right)+{ }^{8 s_{i}} \llbracket e^{\prime} \rrbracket_{\text {Expr }}^{\natural, s_{i}} \sigma$ where $\sigma^{s_{i}}\left(a_{j}\right)=b i n^{8 s_{i}}\left(v_{j}\right)$. By definition of $\operatorname{bin}^{8 s}, \operatorname{bin}^{8 s}(n) *^{8 s_{i}} \operatorname{bin}^{8 s_{i}}\left(v_{j}\right)=\left(\left(n \bmod 2^{8 s_{i}}\right) *\left(v_{j} \bmod 2^{8 s_{i}}\right)\right) \bmod 2^{8 s_{i}}=$ $\left(n * v_{j}\right) \bmod 2^{8 s_{i}}$, c.f. [7, page 42]. Similarly, $\left(n * v_{j}\right) \bmod 2^{8 s_{i}}+{ }^{8 s_{i}} \llbracket e^{\prime} \rrbracket_{\mathrm{Expr}}^{\natural, s_{i}} \sigma=$ $\left(n * v_{j}\right) \bmod 2^{8 s_{i}}+{ }^{8 s_{i}} \operatorname{bin}^{8 s_{i}}\left(\boldsymbol{c}^{\prime} \cdot \boldsymbol{v}+d^{\prime}\right)=\left(n * v_{j}+\boldsymbol{c}^{\prime} \cdot \boldsymbol{v}+d^{\prime}\right) \bmod 2^{8 s_{i}}$. Thus, set $d=d^{\prime}$ and $\left\langle c_{1}, \ldots c_{n}\right\rangle=\left\langle c_{1}^{\prime}, \ldots c_{i-1}^{\prime}, c_{i}^{\prime}+n, c_{i+1}^{\prime}, \ldots c_{n}^{\prime}\right\rangle$ where $c=\left\langle c_{1}, \ldots c_{n}\right\rangle$ and $\boldsymbol{c}^{\prime}=\left\langle c_{1}^{\prime}, \ldots, c_{n}^{\prime}\right\rangle$. Hence $e \equiv \boldsymbol{c} \cdot \boldsymbol{x}+d$.

The force of the above result is that a linear expression $\langle$ Expr $\rangle$ over finite integer variables can be interpreted as an expression over polyhedral variables without regard for overflows or evaluation order. A prerequisite for this convenience is that all variables occurring in an expression have the same size $s$. In contrast, assignments between different sized variables have to revert to a cast statement. In this case, and in the case of conditionals, wrapping has to be made explicit which is the topic of the next section. 


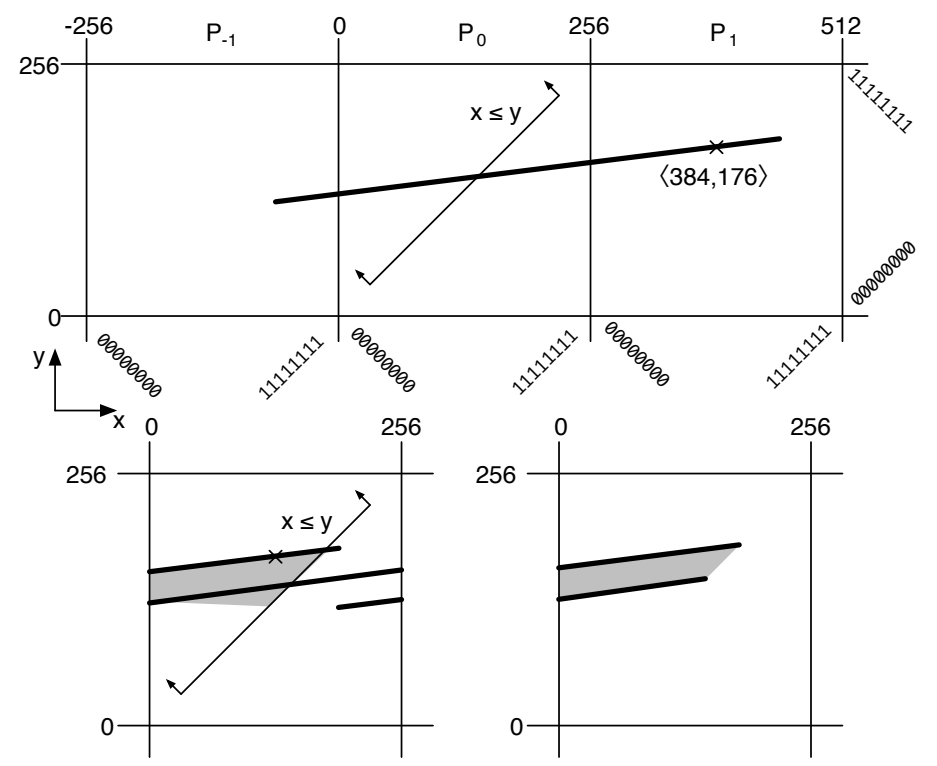

Fig. 4. Explicitly wrapping the possible values of $x$ to its admissible range.

\section{Explicit Wrapping of Polyhedral Variables}

A consequence of the wrapping behaviour of $\gamma_{\boldsymbol{a}}^{\boldsymbol{s}}$ is that the effect of a guard such as $\mathrm{x}<=\mathrm{y}$ cannot be modelled as a transformation from a polyhedron $P$ to $P \sqcap \llbracket x \leq y \rrbracket$. This section explains this problem, discusses possible solutions and proposes an efficient wrapping algorithm wrap.

\subsection{Wrapping Variables with a Finite Range}

In order to illustrate the requirements on the wrap function, consider Figure 4. The thick line in the upper graph denotes $P=\llbracket x+1024=8 y,-64 \leq x \leq 448 \rrbracket$ which we suppose feeds into the guard $\mathrm{x}<=\mathrm{y}$ where $\mathrm{x}$ and $\mathrm{y}$ both represent variables of type uint8. In order to illustrate a peculiarity of modelling the guard, consider the point $\langle x, y\rangle=\langle 384,176\rangle \in P$ and let $\sigma \in \gamma_{\boldsymbol{a}}^{\boldsymbol{s}}(\{\langle 384,176\rangle\})$. Due to implicit wrapping in $\gamma_{\boldsymbol{a}}^{\boldsymbol{s}}$, the state $\sigma$ stipulates that $v a l^{8, \text { uint }}\left(\sigma^{1}\left(a d d r^{\natural}(x)\right)\right)=128$ and $v a l^{8, \text { uint }}\left(\sigma^{1}\left(a d d r^{\natural}(y)\right)\right)=176$. Thus, although $\mathrm{x}<=\mathrm{y}$ is true when interpreting $\mathrm{x}$ and $\mathrm{y}$ as uint8 in $\sigma$, the polyhedron $\{\langle 384,176\rangle\} \sqcap \llbracket x \leq y \rrbracket$ is empty. Hence, it is not correct to model the guard in the classic way as $P \sqcap \llbracket x \leq y \rrbracket$.

In order to model relational tests correctly, the values of expressions occurring on each side of a relational operator have to be wrapped to the type prescribed in an $\mathcal{L}$ (ELang) conditional. In the example, the expression $y$ is already in the required range $[0,255]$ whereas the range of $x$ impinges on the two neighbouring quadrants as indicated in the upper graph of Figure 4. These quadrants are obtained by partitioning the state $P$ into $P_{-1}=P \sqcap \llbracket-256 \leq x \leq-1 \rrbracket, P_{0}=$ 


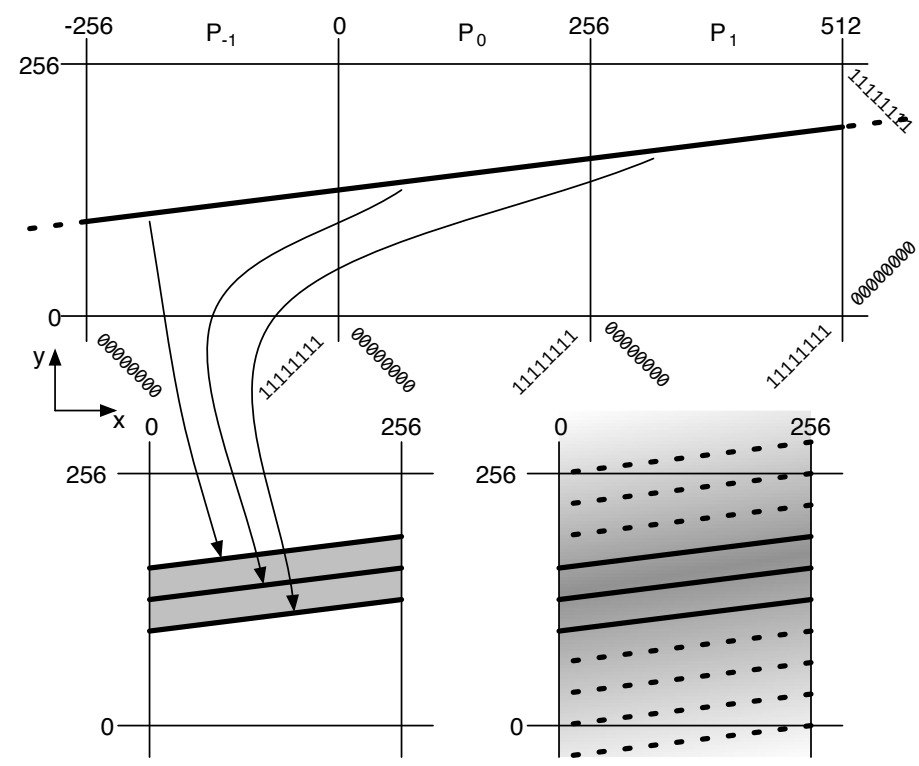

Fig. 5. The quest for an efficient wrapping of unbounded variables.

$P \sqcap \llbracket 0 \leq x \leq 255 \rrbracket$ and $P_{1}=P \sqcap \llbracket 256 \leq x \leq 511 \rrbracket$. The result of wrapping $x$ can now be calculated by translating $P_{-1}$ by 256 units towards positive $x$ coordinates and $P_{1}$ by 256 units towards negative $x$-coordinates, yielding $P^{\prime}=$ $P_{0} \sqcup\left(P_{-1} \triangleright x:=x+256\right) \sqcup\left(P_{1} \triangleright x:=x-256\right)$. The contribution of each partition is shown as a thick line in the lower left graph and the grey region depicts $P^{\prime} \sqcap \llbracket x \leq y \rrbracket$. Observe that a more precise state $P^{\prime \prime}$ can be obtained by intersecting each translated state separately with $\llbracket x \leq y \rrbracket$, that is, by calculating $\left(P_{0} \sqcap \llbracket x \leq y \rrbracket\right) \sqcup\left(\left(P_{-1} \triangleright x:=x+256\right) \sqcap \llbracket x \leq y \rrbracket\right) \sqcup\left(\left(P_{1} \triangleright x:=x-256\right) \sqcap \llbracket x \leq y \rrbracket\right)$. This state, depicted as the grey area in the lower right graph, is smaller than $P^{\prime}$ since $P_{-1}$ does not contribute at all. Indeed, this example shows that polyhedra are not meet-distributive, that is, $P \sqcap\left(P_{1} \sqcup P_{2}\right) \neq\left(P \sqcap P_{1}\right) \sqcup\left(P \sqcap P_{2}\right)$. In this work, we chose to calculate the equivalent of $P^{\prime}$ in our wrapping function wrap as it simplifies the presentation; implementing the refined model is mere engineering.

\subsection{Wrapping Variables with Infinite Ranges}

In the given example, it was possible to obtain a wrapped representation of the values of $x$ and $y$ by calculating the join of three constituent state spaces. In general, however, wrapping $x$ and $y$ can require the join of an infinite number of constituent state spaces as depicted in Figure 5. Here, the line in the upper graph depicts $P=\llbracket x+1024=8 y \rrbracket$, that is, $P$ denotes the same linear relation as before, except that $x$ is unbounded. Translating $P$ by $i$ times the range of uint8 yields $P_{i}=\left(P \triangleright x:=x+i 2^{8} \sqcap \llbracket 0 \leq x \leq 255 \rrbracket\right) \sqcup\left(P \triangleright x:=x-i 2^{8} \sqcap \llbracket 0 \leq x \leq 255 \rrbracket\right)$ for $i \geq 0$. A polyhedron that includes the sequence $P_{j}^{\prime}=\bigsqcup_{0 \leq i \leq j} P_{i}$ can be 


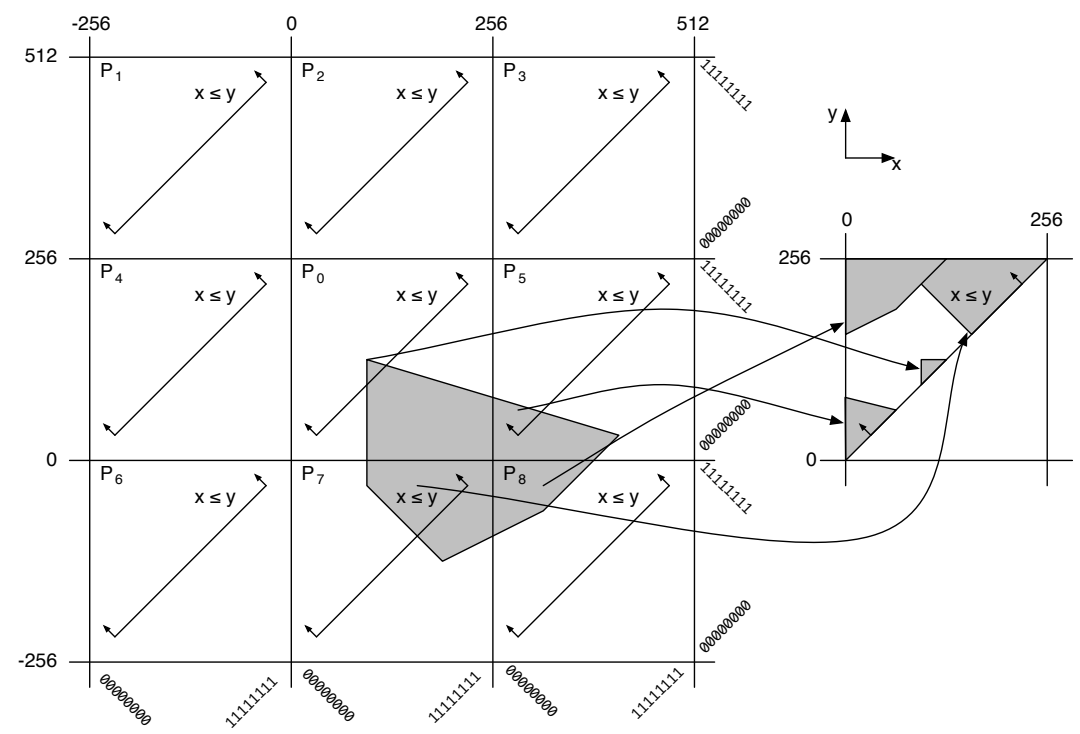

Fig. 6. Precise wrapping of two bounded variables.

computed using widening [6], thereby yielding the grey area in the lower right graph. In fact, this region is equivalent to $\exists_{x}(P) \sqcap \llbracket 0 \leq x \leq 255 \rrbracket$ as it contains neither bounds on $x$ nor relational information between $x$ and other variables. This suggests that, rather than wrapping unbounded variables, it is cheaper and as precise to set them to the whole range of their type. After wrapping $x$, it becomes apparent that $y$ is unbounded too, and hence needs wrapping.

\subsection{Wrapping Several Variables}

Even though the guard $\mathrm{x}<=\mathrm{y}$ used in the example of Section 5.1 involves two variables, it was only necessary to wrap $x$ to obtain a wrapped representation of both $x$ and $y$. The example of Section 5.2 hints at the fact that both variables might need wrapping to ensure that both sides of the guard are within range. In particular, it is not possible to translate a guard $\mathrm{x}<=\mathrm{y}$ to the inequality $x-y \leq 0$ and to merely wrap $x-y$ to $[0,255]$. To illustrate this, consider the simpler case $\mathrm{x}<=42$ which is satisfied for bit sequences of $\mathrm{x}$ that fall within $[0,42]$. In order to evaluate $\mathrm{x}<=42$, set $x^{\prime}=x-42$ and wrap $x^{\prime}$ such that $0 \leq x^{\prime} \leq 255$. The intersection with $\llbracket x^{\prime} \leq 0 \rrbracket$ constraints $x^{\prime}$ to 0 which implies $x=42$ instead of $x \in$ $[0,42]$. Thus, both arguments to a guard $\mathrm{x}<=\mathrm{y}$ need to be wrapped independently.

The example in Figure 5 showed how wrapping the unbounded $x$ leaves $y$ unconstrained which thus has to be wrapped as well. Figure 6 shows a potentially more precise solution for bounded variables in which variables are wrapped simultaneously. Here, the bounded state space shown in grey expands beyond the state $P_{0}$ that corresponds to the actual range of the variables. The result of 


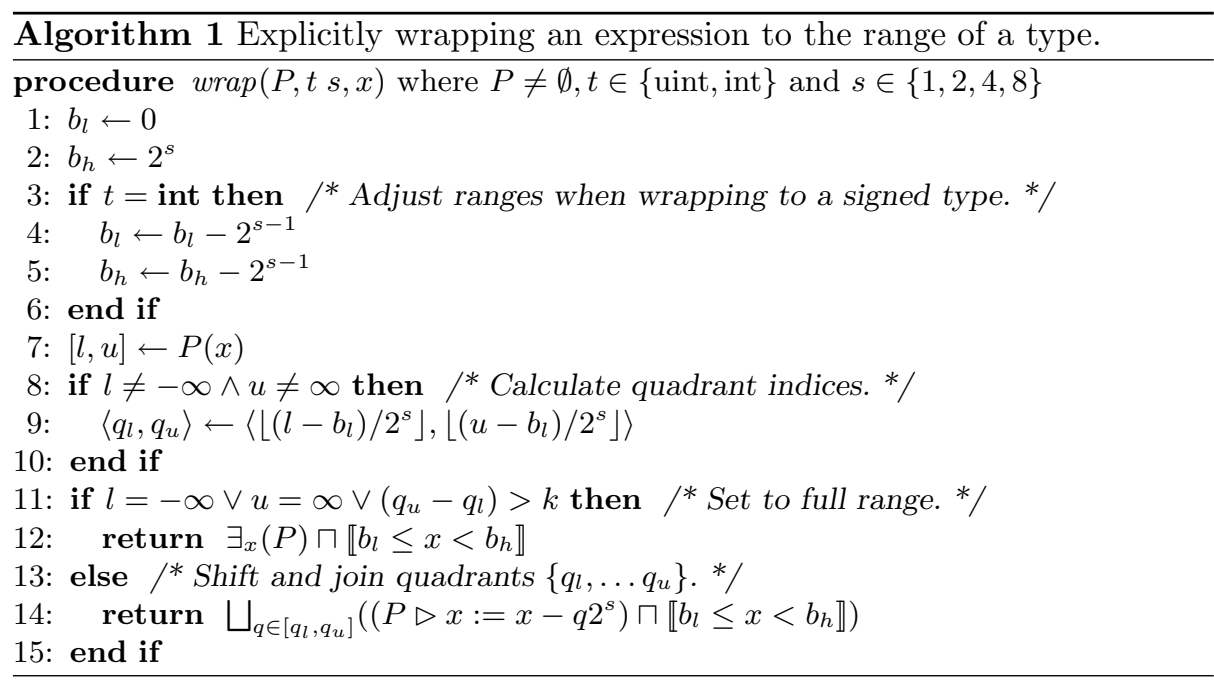

translating each neighbouring quadrant and intersecting it with $x \leq y$ is shown in the graph on the right. Note that the join of these four translated spaces retains no information on either $x$ or $y$. While it is possible that relational information with other variables is retained, wrapping the variables independently has the same precision if one of the variables is within bounds and, in particular, if a variable is compared to a constant. In the 3000 LOC program qmail-smtp that our analysis targets, 427 out of 522 conditionals test a variable against a constant, which motivates our design choice of wrapping variables independently.

\subsection{An Algorithm for Explicit Wrapping}

Guided by the observations made in the three examples, Algorithm 1 gives a procedure to wrap a polyhedral variable to the range of a given integer type. Due to the observations in the last section, we only present an algorithm to wrap one variable at a time. Thus, both sides of a guard have to be wrapped individually.

The algorithm commences by calculating the bounds of the type $t s$. A uint8 type, for instance, will set $b_{l}=0$ and $b_{h}=2^{8}=256$ while an int8 type results in the bounds $b_{l}=0-2^{8-1}=-128$ and $b_{h}=2^{8}-2^{8-1}=128$. Line 7 calculates the bounds of $x$ in $P$. If one of these bounds is infinite, line 12 removes all information on $x$ and restrains $x$ to $\left[b_{l}, b_{h}-1\right]$. In case of finite bounds, line 9 calculates the smallest and largest quadrant into which the values of $x$ impinge. For instance, in the example of Figure 4, these numbers are $q_{l}=-1$ (for the quadrant $[-256,-1]$ ) and $q_{h}=1$ (for $[256,511]$ ). Line 11 ensures that the linear expression is simply set to its maximum bounds if more than $k$ quadrants have to be transposed and joined, where $k$ is a limit that can be tuned to the required precision. Line 14 transposes each quadrant and restricts it to the bounds of the type. The correctness of wrap is asserted below: 
Proposition 2. Given $P \neq \emptyset$ and $P^{\prime}=w r a p\left(P, t s, x_{i}\right)$, the interval $P^{\prime}\left(x_{i}\right)$ lies in the range of the type $t s$. Furthermore $\gamma_{\boldsymbol{a}}^{\boldsymbol{s}}(P) \subseteq \gamma_{\boldsymbol{a}}^{\boldsymbol{s}}\left(P^{\prime}\right)$.

Proof. Upon return from lines 12 and $14, x_{i}$ is restricted to lie between the bounds $b_{l}$ and $b_{h}-1$ of the type $t s$, hence $P^{\prime}\left(x_{i}\right)$ lies in the range of type $t s$.

Suppose $\boldsymbol{a}=\left\langle a_{1}, \ldots a_{n}\right\rangle, \boldsymbol{s}=\left\langle s_{1}, \ldots s_{n}\right\rangle$ where $a_{i}=a d d r^{\natural}\left(x_{i}\right)$ and $s_{i}$ denotes the size of $x_{i}$ in bytes. Let $\sigma \in \gamma_{\boldsymbol{a}}^{\boldsymbol{s}}(P)$. Then there exists $\left\langle v_{1}, \ldots v_{n}\right\rangle \in P \cap \mathbb{Z}^{n}$ such that $\sigma \in \gamma_{\boldsymbol{a}}^{\boldsymbol{s}}(\{\boldsymbol{v}\})$. We consider two behaviours of wrap:

- Suppose that wrap is exited at line 12 . Observe that for any $\boldsymbol{b} \in \mathbb{B}^{8 s_{i}}$ there exists $v \in\left\{b_{l}, \ldots, b_{h}-1\right\}$ such that $\operatorname{bin}^{8 s_{i}}(v)=\boldsymbol{b}$. Hence, there exists $\boldsymbol{v}^{\prime}=$ $\left\langle v_{1}, \ldots v_{i}^{\prime}, \ldots v_{n}\right\rangle$ with $v_{i}^{\prime} \in\left[b_{l}, b_{h}-1\right] \cap \mathbb{Z}$ and $\operatorname{bin}^{8 s_{i}}\left(v_{i}^{\prime}\right)=\operatorname{bin}^{8 s_{i}}\left(v_{i}\right)$. Observe that $\boldsymbol{v}^{\prime} \in P^{\prime}=\exists_{x}(P) \sqcap \llbracket b_{l} \leq x<b_{h} \rrbracket$ and $\boldsymbol{v}^{\prime} \in \mathbb{Z}^{n}$. Hence bits $\boldsymbol{s}_{\boldsymbol{a}}^{\boldsymbol{s}}\left(v_{j}\right)=$ bits $_{\boldsymbol{a}}^{\boldsymbol{s}}\left(v_{j}^{\prime}\right)$ for all $j \in[1, n]$ and it follows that $\sigma \in \gamma_{\boldsymbol{a}}^{\boldsymbol{s}}\left(P^{\prime}\right)$.

- Suppose now that wrap exits at line 14 . Observe that $v_{i} \in[l, u]$ hence there exists $q \in\left[q_{l}, q_{u}\right]$ such that $v_{i}-q 2^{s_{1}} \in\left[b_{l}, b_{h}-1\right]$. Hence, there exists $\boldsymbol{v}^{\prime}=$ $\left\langle v_{1}, \ldots v_{i}^{\prime}, \ldots v_{n}\right\rangle \in P^{\prime}$ such that $v_{i}^{\prime}=v_{i}-q 2^{s_{i}}$. Since $\operatorname{bin}^{8 s_{i}}\left(q 2^{s_{i}}\right)=\mathbf{0}$ it follows that $\operatorname{bin}^{8 s_{i}}\left(v_{i}^{\prime}\right)=\operatorname{bin}^{8 s_{i}}\left(v_{i}-q 2^{s_{i}}\right)=\operatorname{bin}^{8 s_{i}}\left(v_{i}\right)$. Thus $\sigma \in \gamma_{\boldsymbol{a}}^{\boldsymbol{s}}\left(P^{\prime}\right)$.

Note the translation of quadrants using $P \triangleright x:=x+q 2^{s}$ can be implemented by a potentially cheaper affine transformation [2]. However, the shown solution can be readily implemented using other polyhedral domains $[14,16]$ that do not directly support affine translations.

\section{$6 \quad$ An Abstract Semantics for $\mathcal{L}$ (ELang)}

This section defines the abstract semantics of $\mathcal{L}$ (ELang) in which a single polyhedron $P$ is calculated for each label $l$ where each label marks the beginning of a basic block. Starting with the unrestricted polyhedron $\mathbb{R}^{|X|}$ for the first basic block and with the empty polyhedron $\emptyset \subseteq \mathbb{R}^{|X|}$ for all others, the semantic function of the basic blocks are repeatedly evaluated until a (post-)fixpoint is reached [4]. We omit a formal definition of this fixpoint for simplicity. Once a fixpoint is reached, each state $\sigma$ that may arise in the concrete program at $l$ satisfies $\sigma \in \gamma_{\boldsymbol{a}}^{\boldsymbol{s}}(P)$ where $P$ is the polyhedron associated with the label $l$.

The first rule in Figure 7 specifies how the evaluation of statements feeds into the evaluation of control-flow statements. Specifically, 【lookupNext $(l) \rrbracket_{\text {Next }}^{\sharp} P_{l}$ yields tuples such as $\left\langle P_{l^{\prime}}^{\prime}, l^{\prime}\right\rangle$ indicating that $P_{l^{\prime}}^{\prime}$ must be joined with the existing state $P_{l^{\prime}}$ at $l^{\prime}$. For instance, jump $l$ merely returns the current state paired with the target label. The conditional calculates two new polyhedra $P^{\text {then }}$ (which is returned for the label $l$ ) and $P^{\text {else }}$ (which is used to evaluate other control-flow instructions). The calculation of $P^{\text {else }}$ makes use of a function neg which negates a relational operator, for example, neg $\left({ }^{\prime}<^{\prime}\right)={ }^{\prime} \geq^{\prime}$. The auxiliary function cond wraps the two arguments of the relational operator op. Like wrap, this function can only wrap single polyhedral variables which requires that exp is assigned to a temporary variable $y$ which is projected out once the guard is applied.

Observe that enforcing the guard by intersecting with $\llbracket x$ op $y \rrbracket$ has the same effect as wrapping the expression $\exp$ itself since $y=\exp$ holds in $P^{\prime}$. However, 
if wrap returns from line 12 in Algorithm 1, the variable $y$ is merely set to the bounds of the type. In this case wrap discards the relational information between $y$ and $\exp$ and the intersection with $\llbracket x$ op $y \rrbracket$ has no effect on $P^{\prime \prime}$, thereby ignoring the condition. An alternative treatment for expressions $\exp$ that exceed $k$ quadrants would be to discard any previous information on variables in exp using projection and to modify wrap to intersect $P$ with $\llbracket b_{l} \leq \exp <b_{h} \rrbracket$. In this case the information in the guard could be retained by intersecting with $\llbracket x$ op exp $\rrbracket$ at the cost of discarding any previous bounds on the variables of exp.

The following Proposition states the correctness of the conditional:

Proposition 3. If $\sigma \in \gamma_{\boldsymbol{a}}^{\boldsymbol{s}}(P)$ and $v a l^{8 s, t}\left(\sigma^{s}\left(a d d r^{\natural}\left(x_{i}\right)\right)\right)$ op $v a l^{8 s, t}\left(\llbracket \exp \rrbracket_{\operatorname{Expr}}^{\natural, s} \sigma\right)$ then $\left\langle P^{\prime}, l\right\rangle \in \llbracket$ if $t s x_{i}$ op exp then jump $l ; n x t \rrbracket_{\text {Next }}^{\sharp} P$ and $\sigma \in \gamma_{\boldsymbol{a}}^{\boldsymbol{s}}\left(P^{\prime}\right)$.

Proof. Since $\sigma \in \gamma_{\boldsymbol{a}}^{\boldsymbol{s}}(P)$ there exists $\boldsymbol{v} \in P \cap \mathbb{Z}^{n}$ such that $\sigma \in \gamma_{\boldsymbol{a}}^{\boldsymbol{s}}(\{\boldsymbol{v}\})$. Let $\hat{P}=\operatorname{cond}\left(\bar{P}, t s, x_{i}, y, o p\right)$ where $\bar{P}=P \triangleright y:=\llbracket \exp \rrbracket_{\text {Expr }}^{\sharp}$ Then $\left\langle v_{1}, \ldots v_{n}, \hat{v}\right\rangle \in$ $\bar{P} \cap \mathbb{Z}^{n+1}$ where $\left\langle v_{1}, \ldots v_{n}\right\rangle=\boldsymbol{v}, \hat{v}=\boldsymbol{c} \cdot \boldsymbol{v}+d$ and $\exp \equiv \boldsymbol{c} \cdot \boldsymbol{x}+d$. By Proposition 2, there exists $\boldsymbol{v}^{\prime}=\left\langle v_{1}, \ldots v_{i-1}, v_{i}^{\prime}, v_{i+1}, \ldots v_{n}, \hat{v}^{\prime}\right\rangle \in \hat{P} \cap \mathbb{Z}^{n+1}$ such that $\operatorname{bin}^{8 s}\left(v_{i}\right)=\operatorname{bin}^{8 s}\left(v_{i}^{\prime}\right)=\sigma^{8 s}\left(a d d r^{\natural}\left(x_{i}\right)\right)$. By following Proposition 1, $\operatorname{bin}^{8 s}(\hat{v})=\operatorname{bin}^{8 s}\left(\hat{v}^{\prime}\right)=\llbracket \exp \rrbracket_{\text {Expr }}^{\natural, s}$. Furthermore, $v_{i}^{\prime}$ and $\hat{v}^{\prime}$ lie in the range of $t s$ and thus $v a l^{8 s, t}\left(\sigma^{8 s}\left(a d d r^{\natural}\left(x_{i}\right)\right)\right)=v_{i}^{\prime}$ and $v a l^{8 s, t}\left(\llbracket \exp \rrbracket_{\operatorname{Expr}}^{\natural, s} \sigma\right)=\hat{v}^{\prime}$. Hence $\boldsymbol{v}^{\prime} \in \hat{P} \sqcap \llbracket x$ op $y \rrbracket$ for op $\notin\{\neq\}$. With $P^{\prime}=\exists_{y}(\hat{P} \sqcap \llbracket x$ op $y \rrbracket)$ it follows $\sigma \in \gamma_{\boldsymbol{a}}^{\boldsymbol{s}}\left(P^{\prime}\right)$. The argument is similar for op $\in\{\neq\}$.

The fall-through case can be shown correct by a similar argument.

Due to the modulo nature of $\gamma_{\boldsymbol{a}}^{\boldsymbol{s}}$ the evaluation of linear expressions and assignments resembles that of classic polyhedral analysis in that linear expressions in the program are simply re-interpreted as expressions over polyhedra variables. This holds true even for casts between different sized variables as long as the target variable is smaller. Assigning smaller variables to larger, on the contrary, requires that wrapping is made explicit since a value that exceeds the range of the smaller source variable would wrap in the actual program whereas it might not exceed the range of the larger target variable.

We conclude this section with a correctness argument for the cast statement:

Proposition 4. Suppose $\sigma \in \gamma_{\boldsymbol{a}}^{\boldsymbol{s}}(P), \sigma^{\prime}=\llbracket s_{1} x_{i}=t s_{2} x_{j} \rrbracket_{\text {Stmt }}^{\natural} \sigma$ and let $P^{\prime}=\llbracket s_{1} x_{i}=t s_{2} x_{j} \rrbracket_{\text {Stmt }}^{\sharp} P$. Then $\sigma^{\prime} \in \gamma_{\boldsymbol{a}}^{\boldsymbol{s}}\left(P^{\prime}\right)$.

Proof. Since $\sigma \in \gamma_{\boldsymbol{a}}^{\boldsymbol{s}}(P)$ there exists $\boldsymbol{v}=\left\langle v_{1}, \ldots v_{n}\right\rangle \in P$ such that $\sigma \in \gamma_{\boldsymbol{a}}^{\boldsymbol{s}}(\{\boldsymbol{v}\})$. Let $\left\langle v_{1}^{\prime}, \ldots v_{n}^{\prime}\right\rangle \in P \triangleright x_{i}:=x_{j}$ where $v_{i}^{\prime}=v_{j}^{\prime}$ and $v_{k}^{\prime}=v_{k}$ for all $k \neq i$. By Lemma $1, \sigma^{s_{k}}\left(a d d r^{\natural}\left(x_{k}\right)\right)=\sigma^{s_{k}}\left(a d d r^{\natural}\left(x_{k}\right)\right)$ for all $k \neq i$. By definition of $\llbracket \cdot \rrbracket_{\text {Stmt }}^{\natural}$, we need to show that $\sigma^{s_{1}}\left(a d d r^{\natural}\left(x_{i}\right)\right)=\operatorname{bin}^{8 s_{1}}\left(v a l^{8 s_{2}, t}\left(\sigma^{s_{2}}\left(a d d r^{\natural}\left(x_{j}\right)\right)\right)\right)$.

- Suppose $s_{1} \leq s_{2}$. Then $\operatorname{bin}^{8 s_{1}}(x)=\operatorname{bin}^{8 s_{1}}\left(\operatorname{val}^{8 s_{2}, t}\left(\operatorname{bin}^{8 s_{2}}(x)\right)\right)$. But $\operatorname{bin}^{8 s_{1}}\left(v_{i}^{\prime}\right)=$ $\sigma^{s_{1}}\left(a d d r^{\natural}\left(x_{i}\right)\right)$ and $\operatorname{bin}^{8 s_{2}}\left(v_{j}\right)=\sigma^{s_{2}}\left(a d d r^{\natural}\left(x_{j}\right)\right)$, thus $\sigma^{\prime} \in \gamma_{\boldsymbol{a}}^{\boldsymbol{s}}\left(P^{\prime}\right)$ follows.

- Suppose now that $s_{1}>s_{2}$. By Proposition 2 , there exists $\left\langle v_{1}, \ldots \hat{v}_{i}, \ldots v_{n}\right\rangle \in$ $P^{\prime}$ such that $\operatorname{bin}^{8 s_{2}}\left(\hat{v}_{i}\right)=\operatorname{bin}^{8 s_{2}}\left(v_{j}\right)$ and $\hat{v}_{i}$ lies in the range of $t s_{2}$, that is, $\operatorname{val}^{8 s_{2}, t}\left(\operatorname{bin}^{8 s_{2}}\left(\hat{v}_{i}\right)\right)=\hat{v}_{i}$. But since $\operatorname{bin}^{8 s_{2}}\left(\hat{v}_{i}\right)=\operatorname{bin}^{8 s_{2}}\left(v_{j}\right)=\sigma^{\prime s_{1}}\left(\operatorname{addr} r^{\natural}\left(x_{j}\right)\right)$ it follows that $\sigma^{\prime s_{1}}\left(a d d r^{\natural}\left(x_{i}\right)\right)=\operatorname{bin}^{8 s_{1}}\left(\hat{v}_{i}\right)$ as required. 
Basic Blocks.

$\llbracket l: s_{1} ; \ldots s_{n} ; \rrbracket_{\text {Block }}^{\sharp} P=\llbracket \operatorname{lookupNext}(l) \rrbracket_{\text {Next }}^{\sharp}\left(\llbracket s_{n} \rrbracket_{\text {Stmt }}^{\sharp}\left(\ldots \llbracket s_{1} \rrbracket_{\text {Stmt }}^{\sharp} P \ldots\right)\right)$

Control Flow.

$\llbracket$ jump $l \rrbracket_{\text {Next }}^{\sharp} P=\{\langle P, l\rangle\}$

$\llbracket$ if $t$ s $v$ op exp then jump $l ; n x t \rrbracket_{\text {Next }}^{\sharp} P=\left\{\left\langle P^{\text {then }}, l\right\rangle\right\} \cup \llbracket n x t \rrbracket_{\text {Next }}^{\sharp} P^{\text {else }}$

where $P^{\prime}=P \triangleright y:=\llbracket \exp \rrbracket_{\text {Expr }}^{\sharp}$ where $y \in T$ fresh

$P^{\text {then }}=\exists_{y}\left(\operatorname{cond}\left(P^{\prime}, t s, v, y, o p\right)\right)$

$P^{e l s e}=\exists_{y}\left(\operatorname{cond}\left(P^{\prime}, t s, v, y, \operatorname{neg}(o p)\right)\right)$

$\operatorname{cond}(P, t s, x, y, o p)= \begin{cases}\left(P^{\prime \prime} \sqcap \llbracket x<y \rrbracket\right) \sqcup\left(P^{\prime \prime} \sqcap \llbracket x>y \rrbracket\right) & \text { if } o p \in\{\neq\} \\ \left(P^{\prime \prime} \sqcap \llbracket x \text { op } y \rrbracket\right) & \text { otherwise }\end{cases}$

where $P^{\prime}=\operatorname{wrap}(P, t s, x)$

$P^{\prime \prime}=\operatorname{wrap}\left(P^{\prime}, t s, y\right)$

Expressions.

$\llbracket n \rrbracket_{\mathrm{Expr}}^{\sharp}=n$

$\llbracket n * v+\exp \rrbracket_{\mathrm{Expr}}^{\sharp}=n v+\llbracket \exp \rrbracket_{\mathrm{Expr}}^{\sharp}$

Assignments.

$\llbracket s v=\exp \rrbracket_{\mathrm{Stmt}}^{\sharp} P=P \triangleright v:=\llbracket \exp \rrbracket_{\mathrm{Expr}}^{\sharp}$

Type Casts.

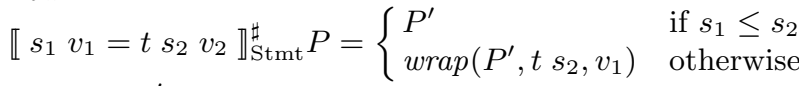

where $P^{\prime}=P \triangleright v_{1}:=v_{2}$

Fig. 7. Abstract semantics of $\mathcal{L}$ (ELang).

\section{Discussion}

The existence of a concretisation map $\gamma_{\boldsymbol{a}}^{\boldsymbol{s}}$ begs the question of whether an abstraction map can be defined. For classic polyhedral analysis [6], it is well-known that no best abstraction exists for certain shapes such as a disc [4]. In the context of our analysis, the set of concrete states $\Sigma$ is finite. However, a given set of states still has no best abstraction. Consider $\sigma \in \Sigma$ with $\sigma^{1}\left(\operatorname{addr} r^{\natural}(\mathrm{x})\right)=11111111$, $P_{1}=\llbracket x=-1 \rrbracket$ and $P_{2}=\llbracket x=255 \rrbracket$. Although $\sigma \in \gamma_{\boldsymbol{a}}^{\boldsymbol{s}}\left(P_{1}\right)=\gamma_{\boldsymbol{a}}^{\boldsymbol{s}}\left(P_{2}\right), P_{1}$ and $P_{2}$ are incomparable. As a consequence, the meet operation can only be applied after wrap has expressed the polyhedra in the same quadrant and thereby made them comparable. Termination is not compromised as wrap is monotonic.

Since different polyhedra can describe the same set of concrete states, care is needed when applying join. Suppose that the loop in Figure 8 is entered with $P=\llbracket x=-1 \rrbracket$ : the largest value an unsigned variable can take. As the loop invariant $x \geq 42$ mentions $x, Q=P \sqcup U$ is wrapped to $R=\operatorname{wrap}(Q, \operatorname{uint8}, x)=$ $\llbracket x=255 \rrbracket$. A precision loss occurs when $P$ and $U=\llbracket x=254 \rrbracket$ are joined to obtain 


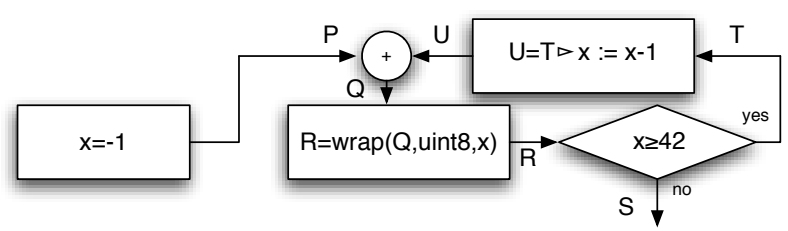

Fig. 8. Precision loss incurred by joining flow paths.

$\llbracket-1 \leq x \leq 254 \rrbracket$ as $x$ cannot fall below 42 . One solution to this particular problem is to unroll the loop once, which avoids the join of different representatives.

Observe that wrap is idempotent and, as such, is the identity if the variable is in range. An important consequence is that our solution is as precise as classic polyhedral analysis if all variables remain within the range of their types.

An interesting benefit of $\gamma_{\boldsymbol{a}}^{\boldsymbol{s}}$ is that the possible values of a byte $x$ can be represented as either $\llbracket-128 \leq x \leq 127 \rrbracket$ or $\llbracket 0 \leq x \leq 255 \rrbracket$. For example, an analysis of $\mathrm{C}$ string buffers [13] does not model individual array elements but tracks a single NUL position (a character with value zero) within the array. Even though char is often signed, the range $[0,255]$ can be returned when reading a byte from the array which can then be refined using the NUL position to $[1,255]$ whenever the access lies in front of the NuL position (see [15] for an example). If a signed range had to be returned, it would include the NUL character since $[-128,-1] \cup[1,127]=[-128,127]$ is the best convex approximation. Without this tactic, it can be difficult to prove termination of loops that iterate over strings.

\section{Related Work and Conclusion}

A sound analysis must reason about overflowing calculations and correctly model conversions between unsigned and signed integers. Rather than contaminating an analysis with the low-level details of two's complement wrapping behaviour, we presented a computationally light-weight solution based on a novel concretization map for polyhedra which eliminates the need to check for wrapping in assignments of linear expression and conversions to smaller integers. Conditionals and other casts require an implementation using the presented algorithm wrap. We proved the presented analysis correct and argued the precision is no worse than that of classic polyhedral analysis that warns about every wrapping.

Although a number of works have addressed the modulo properties of congruences [1,10-12], little work exists for polyhedral analyses. Blanchet et al. use a two-tier approach [5]: For signed integers, any wrapping is erroneous. In this case, each time a variable is set, its range is checked for overflows. Overflows of unsigned integers are assumed to be intentional as wrapping may result from bit-level operations. This approach requires a separation of signed and unsigned variables which incurs false warnings for many programs, such as the $\mathrm{C}$ program in our introduction. Indeed, an analysis of our example would lead to the misleading warning about converting from a signed to an unsigned integer.

Acknowledgements. This work is supported by EPSRC project EP/C015517. 


\section{References}

1. R. Bagnara, K. Dobson, P. M. Hill, M. Mundell, and E. Zaffanella. Grids: A Domain for Analyzing the Distribution of Numerical values. In G. Puebla, editor, LOPSTR, volume 4407 of LNCS, Venice, Italy, 2007. Springer. To appear.

2. R. Bagnara, E. Ricci, E. Zaffanella, and P. M. Hill. Possibly Not Closed Convex Polyhedra and the Parma Polyhedra Library. In M. V. Hermenegildo and G. Puebla, editors, Static Analysis Symposium, volume 2477 of LNCS, pages 213229, Madrid, Spain, 2002. Springer.

3. Joint Technical Committee. International Standard ISO/IEC of C 98/99, 1999.

4. P. Cousot and R. Cousot. Comparing the Galois Connection and Widening/Narrowing Approaches to Abstract Interpretation. In M. Bruynooghe and M. Wirsing, editors, Programming Language Implementation and Logic Programming, volume 631 of $L N C S$, pages 269-295. Springer, 1992.

5. P. Cousot, R. Cousot, J. Feret, L. Mauborgne, A. Miné, D. Monniaux, and X. Rival. The ASTRÉE Analyzer. In European Symposium on Programming, pages 21-30, Edinburgh, Scotland, 2005. Springer.

6. P. Cousot and N. Halbwachs. Automatic Discovery of Linear Constraints among Variables of a Program. In Principles of Programming Languages, pages 84-97, Tucson, Arizona, 1978. ACM Press.

7. H. Davenport. The Higher Arithmetic. Cambridge University Press, seventh edition, 1952.

8. N. Dor, M. Rodeh, and M. Sagiv. Cleanness Checking of String Manipulations in C Programs via Integer Analysis. In P. Cousot, editor, Static Analysis Symposium, volume 2126 of $L N C S$, pages 194-212, Paris, France, 2001. Springer.

9. N. Dor, M. Rodeh, and M. Sagiv. CSSV: Towards a Realistic Tool for Statically Detecting All Buffer Overflows in C. In R. Gupta, editor, Programming Language Design and Implementation, pages 155-167, San Diego, USA, June 2003. ACM Press.

10. P. Granger. Static Analysis of Arithmetic Congruences. International Journal of Computer Mathematics, 30:165-199, 1989.

11. P. Granger. Static Analyses of Congruence Properties on Rational Numbers (Extended Abstract). In Symposium on Static Analysis, pages 278-292, London, UK, 1997. Springer.

12. Markus Müller-Olm and Helmut Seidl. Analysis of Modular Arithmetic. In European Symposium on Programming, volume 3444 of $L N C S$, pages 46-60. Springer, 2005.

13. A. Simon and A. King. Analyzing String Buffers in C. In Algebraic Methodology and Software Technology, volume 2422 of LNCS, pages 365-379. Springer, 2002.

14. A. Simon and A. King. Exploiting Sparsity in Polyhedral Analysis. In C. Hankin and I. Siveroni, editors, Static Analysis Symposium, volume 3672 of LNCS, pages 336-351. Springer, September 2005.

15. A. Simon and A. King. Widening Polyhedra with Landmarks. In Naoki Kobayashi, editor, Asian Symposium on Programming Languages and Systems, volume 4279 of $L N C S$, pages 166-182. Springer, November 2006.

16. A. Simon, A. King, and J. M. Howe. Two Variables per Linear Inequality as an Abstract Domain. In M. Leuschel, editor, Logic-based Program Synthesis and Transformation, volume 2664 of LNCS, pages 71-89. Springer, September 2003. 